\title{
Diagnostic and Management Errors in an Immunodeficient Patient with Pneumococcal Pneumonia with Bacteraemia due to Incorrect Assessment of the Patient's Immune Status
}

\author{
Takahiro Ito, Yukinori Harada, Taro Shimizu \\ Department of Diagnostic and Generalist Medicine, Dokkyo Medical University Hospital, Mibu, Tochigi, Japan
}

Received: $12 / 04 / 2021$

Accepted: $13 / 04 / 2021$

Published: $26 / 04 / 2021$

How to cite this article: Ito T, Harada Y, Shimizu T. Diagnostic management errors in an immunodeficient patient with pneumococcal pneumonia with bacteraemia due to incorrect assessment of the patient's immune status. EJCRIM 2021;8: doi:10.12890/2021_002557.

Conflicts of Interests: The Authors declare that there are no competing interests.

This article is licensed under a Commons Attribution Non-Commercial 4.0 License

\section{ABSTRACT}

A 79-year-old woman presented with fever and pleural chest pain. Based on the assessment of mild community pneumonia in an immunocompetent patient, outpatient follow-up was planned. However, the patient was admitted several hours later with a diagnosis of pneumococcal pneumonia with bacteraemia. In addition, selective immunoglobulin $\mathrm{M}$ deficiency was detected. In this case, although a history of recurrent osteomyelitis was provided, the physicians overlooked the information suggesting immunodeficiency, which led to an incorrect diagnostic and management decision. Obtaining the past medical history is essential, but utilizing it is even more important to avoid clinical decision-making errors.

\section{LEARNING POINTS}

- Knowing the immune status of patients with infectious disease is vital for accurate clinical decision-making.

- Patients are sometimes unaware of their immunodeficiency despite having signs indicating indolent immunodeficiency, such as a history of recurrent osteomyelitis.

- Inferring and clarifying the immunodeficiency behind the patient's history was the turning point in this diagnosis.

\section{KEYWORDS}

Immunoglobulin M deficiency, osteomyelitis, pneumococcal bacteraemia

\section{CASE DESCRIPTION}

A 79-year-old woman presented to the emergency department of our hospital with a high-grade fever $\left(38.8^{\circ} \mathrm{C}\right)$, chills and left-side chest pain, which had developed 4 hours previously. She had a history of recurrent osteomyelitis (in her 20's), hepatitis C infection (sustained virological response) and chronic kidney disease. The cause of the recurrent osteomyelitis was unknown, and careful examination had not been performed. Her hepatitis $\mathrm{C}$ and chronic kidney disease had been followed up at a specialized department, and had no recent significant changes. There was no history of drinking alcohol or smoking. Her family did not have a history of immunodeficiency. She had never taken the pneumococcal vaccine. 
She was alert and did not seem distressed. On examination, her blood pressure was $154 / 92 \mathrm{mmHg}$, pulse rate was 92 per minute, respiratory rate was 20 per minute, body temperature was $37.2^{\circ} \mathrm{C}$, and oxygen saturation was $97 \%$ on ambient air. Chest auscultation revealed no abnormal breath sounds, respiratory crackles or heart murmurs. There was no nuchal rigidity, joint swelling or abdominal tenderness. The test for coronavirus disease 2019 was negative. At this visit, while her white blood cell counts were slightly increased $\left(12.9 \times 103 / \mathrm{mm}^{3}\right)$, other laboratory findings were unremarkable. Chest and abdominal computed tomography without contrast revealed consolidation in the lung left lower lobe (Fig. 1). She was diagnosed with community-acquired pneumonia. Her general condition was good, so physicians chose to follow her at an outpatient clinic. She received intravenous ceftriaxone and was discharged home with a prescription of oral antibiotics. Outpatient follow-up was planned.

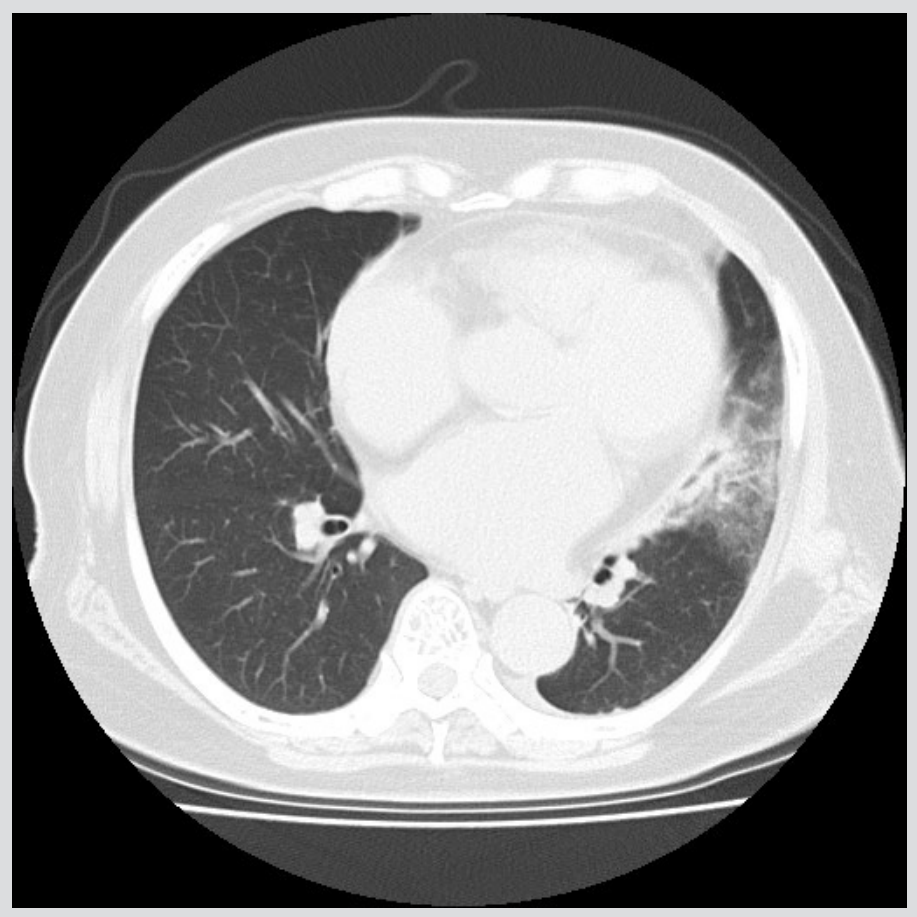

Figure 1. Computed tomography without contrast showing consolidation in the left lung lower lobe

However, 8 hours after discharge, the patient returned to the hospital because blood cultures obtained at presentation turned out to be positive for Streptococcus pneumoniae. At that time, although her condition seemed stable and there were no signs of meningitis or arthritis, the white blood cell count $\left(30.2 \times 103 / \mathrm{mm}^{3}\right)$ and C-reactive protein level $(17 \mathrm{mg} / \mathrm{dl})$ were significantly increased. She was admitted with a diagnosis of pneumococcal pneumonia with bacteraemia and was continued on intravenous ceftriaxone. The following blood culture was sterile, and there was no evidence of endocarditis.

During her admission, investigations for immunodeficiency were conducted based on the history of recurrent osteomyelitis in her 20's and the current pneumococcal bacteraemia, which revealed an isolated low serum level of immunoglobulin (Ig) M (IgM 12.7 mg/dl, IgG 1405 $\mathrm{mg} / \mathrm{dl}$ and IgA $268 \mathrm{mg} / \mathrm{dl}$ ). There was no evidence of hyposplenism because the spleen was a standard size, and no Howell-Jolly bodies were found in the peripheral blood. The antinuclear antibody and anti-double-stranded DNA IgG antibody tests were negative, and there was no evidence of autoimmune disease. Therefore, the patient was clinically diagnosed with primary selective IgM deficiency. She completed 14 days of intravenous ceftriaxone. Her condition rapidly improved, and she was discharged home without complications. Pneumococcal vaccination was planned for an outpatient follow-up visit.

\section{DISCUSSION}

Selective IgM deficiency is a type of immunodeficiency where serum IgM is selectively absent or deficient, defined as a serum lgM level less than 2 standard deviations below the lower reference limit with a normal range of serum IgA and IgG levels ${ }^{[1]}$. Because selective IgM deficiency is under-recognized by physicians and IgM is not routinely checked, the deficiency can be underdiagnosed ${ }^{[2]}$. 
However, physicians should consider selective IgM deficiency as an indolent immunodeficiency when seeing patients who present with a recurrent bacterial infection such as osteomyelitis. Patients with selective IgM deficiency may be asymptomatic; however, approximately $80 \%$ of patients with selective IgM deficiency present with bacterial, viral, fungal or protozoan infection. Recurrent otitis media, chronic sinusitis, bronchitis, bronchiectasis, pneumonia, urinary tract infections, cellulitis, meningitis and sepsis were reported as common infectious diseases in patients with selective IgM deficiency ${ }^{[2]}$.

Selective IgM deficiency is classified into primary and secondary. Secondary selective IgM deficiency is associated with malignancy or several autoimmune diseases including systemic lupus erythematosus, rheumatoid arthritis, Sjogren's syndrome, Hashimoto's thyroiditis, autoimmune haemolytic anaemia, autoimmune glomerulonephritis, coeliac disease and Crohn's disease ${ }^{[3]}$. In the present case, we suspected primary selective IgM deficiency because there were no signs indicating malignancy or autoimmune diseases.

This case underscores that physicians should consider the presence of immunodeficiency when seeing patients who have a history of recurrent severe infections, even if these episodes occurred a long time ago, as in this case ${ }^{[4]}$. The diagnosis or suspicion of immunodeficiency may significantly change the clinical decision-making, particularly decisions related to the management or prevention of infectious disease. For example, in this case, physicians thought of the patient as having mild pneumonia, which could be treated in the outpatient setting even though the patient reported a history of osteomyelitis. However, the physicians would have recommended the patient for admission if they had known that she had immunodeficiency. In addition, the patient had never received pneumococcal vaccination due to a vague fear of vaccines, but decided to receive it once she understood that she was at high risk of life-threatening infectious disease due to her immunodeficiency. Physicians should be aware of patients' past medical history and should try to identify the actual cause of previous illness.

\section{REFERENCES}

Gupta S, Gupta A. Defining primary selective IgM deficiency. J Clin Immunol 2019;39:350-352.

Gupta S, Gupta A. Selective IgM deficiency-an underestimated primary immunodeficiency. Front Immunol 2017;8:1056.

Goldstein MF, Goldstein AL, Dunsky EH, Dvorin DJ, Belecanech GA, Shamir K. Pediatric selective IgM immunodeficiency. Clin Dev Immunol 2008;2008:624850.

Oliveira JB, Fleisher TA. Laboratory evaluation of primary immunodeficiencies. J Allergy Clin Immunol 2010;125:S297-S305. 